\title{
Characterization of Electrons and X-rays Produced using Chirped Laser Pulses in a Laser Wakefield Accelerator
}

\author{
T. Z. Zhao ${ }^{1,2}$, K. Behm ${ }^{1,2}$, Z-H He He $^{1,2}$, A. Maksimchuk ${ }^{1}$, J. A. \\ Nees $^{1}$, V. Yanovsky ${ }^{1}$, A. G. R. Thomas ${ }^{1,2}$, and K. Krushelnick ${ }^{1,2}$ \\ ${ }^{1}$ Center for Ultrafast Optical Science, University of Michigan, Ann Arbor, MI 48109, \\ USA \\ ${ }^{2}$ Nuclear Engineering and Radiological Sciences, University of Michigan, Ann Arbor, \\ MI 48109, USA \\ E-mail: tonyzhao@umich.edu
}

\begin{abstract}
The electron injection process into a plasma-based laser wakefield accelerator can be influenced by modifying the parameters of the driver pulse. We present an experimental study on the combined effect of the laser pulse duration, pulse shape, and frequency chirp on the electron injection and acceleration process and the associated radiation emission for two different gas types - a $97.5 \% \mathrm{He}$ and $2.5 \% \mathrm{~N}_{2}$ mixture and pure He. In general, the shortest pulse duration with minimal frequency chirp produced the highest energy electrons and the most charge. Pulses on the positive chirp side sustained electron injection and produced higher charge, but lower peak energy electrons, compared with negatively chirped pulses. A similar trend was observed for the radiant energy. The relationship between the radiant energy and the electron charge remained linear over a threefold change in the electron density and was independent of the drive pulse characteristics. X-ray spectra showed that ionization injection of electrons into the wakefield generally produced more photons than self injection for all pulse durations/frequency chirp and had less of a spread in the number of photons around the peak X-ray energy.
\end{abstract}

PACS numbers: $\mathrm{X}$

Keywords: X Submitted to: $X$

\section{Introduction}

High quality, quasimonoenergetic electron beams can be generated in a plasma-based laser wakefield accelerator. The electron beams are typically accelerated in the bubble regime in which the ponderomotive force of the laser pulse fully expels the background electrons and leaves behind an ion cavity (bubble) in its wake. Under the appropriate 
conditions, the electrons become trapped inside this cavity and can be accelerated to $\mathrm{GeV}$ energies due to the longitudinal electric field $[1,2,3,4,5]$. The electron beams also undergo oscillations as a result of the transverse focusing electromagnetic field in the cavity. The oscillations result in the emission of synchrotron radiation, with properties determined by a strength parameter $K \equiv r_{\beta} k_{p} \sqrt{\gamma / 2}\left(r_{\beta}\right.$ is the transverse oscillation amplitude, $k_{p} \equiv \omega_{p} / c, \omega_{p}$ is the plasma frequency, and $\gamma$ is the electron relativistic factor), the betatron frequency $\omega_{\beta}=\omega_{p} / \sqrt{2 \gamma}$, and the electron energy $E=\gamma m c^{2}[6]$. Thus, the properties of the emitted radiation are directly coupled to that of the electron beam and optimization of the electron injection process can lead to favorable X-ray characteristics $[7,8]$.

The injection process (and hence, the associated radiation emission) is sensitive to laser pulse characteristics such as its duration, shape, and frequency chirp. The pulse duration generally separates the evolution of the wakefield into three distinct regimes. In the laser wakefield acceleration (LWFA) regime, the pulse duration is shorter than the plasma wavelength and the excitation of the plasma wave (and subsequent electron injection) is most effective. When the pulse duration is on the order of the plasma wavelength, electron injection and acceleration occurs in the "forced"-LWFA regime. In this regime, the drive pulse is compressed by group velocity dispersion and subsequently amplified beyond the wavebreaking limit [9]. Longer pulses leads to the regime of selfmodulated (SM) LWFA, where the instability of the pulse with the plasma frequency drive the wakefield excitation. In both the forced and SM-LWFA regime, it is also possible for the laser field to directly accelerate the electrons [10].

With regard to frequency chirping and pulse shape, theoretical and experimental studies have shown that a positive chirp with a fast-rising leading edge can increase selftrapping of electrons by increasing the wakefield amplitude generated by the chirped pulse. The higher amplitude wakefield then serves to decrease the minimum momentum required to trap electrons $[11,12,13,14,15,16,17,18]$. On the other hand, simulations have shown that incoherently stacking negatively chirped pulses of different wavelengths can be used to create electron beams with energies higher than that obtained using optimally compressed pulses. The negatively chirped pulse can compensate for the red-shifting occurring at the front of the bubble and slow pulse self-steepening. This results in a decrease in the pump depletion occurring at the leading edge of the drive pulse and effectively increases the dephasing length. However, this technique requires a pulse bandwidth, $\Delta \omega$, on the order of the carrier frequency, $\omega_{0}$, and is not feasible for most chirped-pulse amplification (CPA) systems [19]. In these studies, the energy of the electron beam and the resulting X-ray emission have not been experimentally characterized as a function of the drive pulse parameters using a typical Ti:Sapphire CPA system.

In the present work, we investigate the effect of a chirped laser pulse on the electron injection process and the associated radiation emission using a CPA system where $\Delta \omega / \omega_{0} \sim 0.04$. The integrated electron charge and peak energy, along with the radiant energy (i.e., X-ray flux), are characterized as a function of the electron density 
and drive pulse characteristics (i.e., pulse duration, shape, and frequency chirp). In addition, we also compare the X-ray spectra resulting from the betatron oscillations of the electrons inside the wakefield cavity for two different gas types as a function of the pulse characteristics.

\section{Experimental setup}

The experiments were carried out using the $800 \mathrm{~nm}$, Ti:Sapphire HERCULES laser facility at the University of Michigan. Figure 1 shows the setup. The laser beam is focused using an $f / 20$ off-axis parabolic mirror to a vacuum beam waist $w_{0}=26 \mu \mathrm{m}$ (at $1 / e^{2}$ of peak intensity). The energy spectra of the electron beams were measured using an electron spectrometer (ESPEC) which consisted of a $15 \mathrm{~cm}$ long, $0.8 \mathrm{~T}$ dipole magnet in conjunction with a LANEX scintillating detector and a Photometrics CoolSNAP 12bit CCD camera. The ESPEC LANEX also provided information on the total electron charge for each shot. Radiant energy was measured using an Andor iKon-M BR-DD camera placed $2.5 \mathrm{~m}$ downstream from the interaction region. The X-ray camera was shielded from the laser light using a $50 \mu \mathrm{m}$ Be window. A $2 \mu \mathrm{m}$ thick nitrocellulose pellicle reflected $4 \%$ of the main beam and was used to probe the gas cell in the transverse direction before entering a shearing Michelson interferometer, thus allowing electron density measurements to be taken. The use of microscope glass slides in place of the printed plastic walls allowed the probe beam access inside the gas cell.

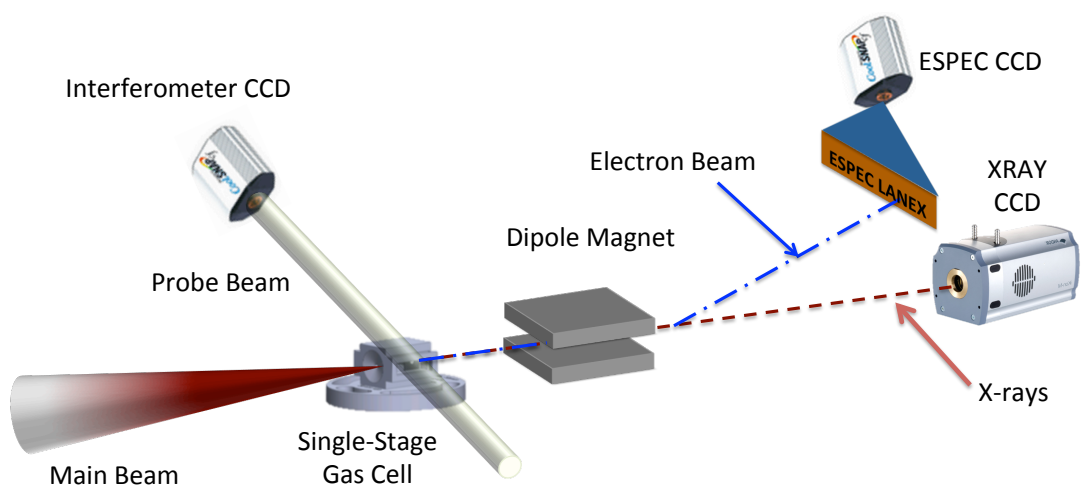

Figure 1. (Color online) Schematic of the experimental setup. The main beam is focused using an $f / 20$ off-axis paraboloid onto a single-stage gas cell filled with either a mixed gas $\left(97.5 \% \mathrm{He}\right.$ and $\left.2.5 \% \mathrm{~N}_{2}\right)$ or pure He. Electrons exiting the cell were swept by a dipole magnet and imaged using the ESPEC diagnostic. The radiant energy was measured $2.5 \mathrm{~m}$ downstream using an Andor CCD camera. 
Measurements of the time-dependent intensity and phase of the laser pulse were conducted using second-order frequency-resolved optical grating (FROG) based on second harmonic generation ( $\mathrm{SHG}$ ) with a sub-Joule beam from the initial regenerative amplifier. This allows the pulse shape to be uniquely determined by using a multiparametric fitting algorithm [20]. Slight asymmetries in the experimentally measured SHG FROG traces can occur along the time axis due to a combination of spatial chirp and pulse front tilt. In order to analyze a larger portion of the measurements, the traces were symmetrized using an average of the left and right portions of the trace. The compressor grating separation was varied to obtain the shortest root-mean-square (RMS) pulse duration with minimal frequency chirp. This grating position is referred to as the "compressor zero". The duration/frequency chirp of the pulse was then simultaneously varied by changing the separation of the compressor gratings. Decreasing (increasing) the separation of the compressor gratings imparted a positive (negative) frequency chirp as well as stretching the pulse and changing the slope of its leading edge. As the pulse was stretched, its energy remained constant. Figure 2 shows typical intensity profiles for chirped and optimally compressed pulses and the pulse duration as a function of the relative grating separation from compressor zero.
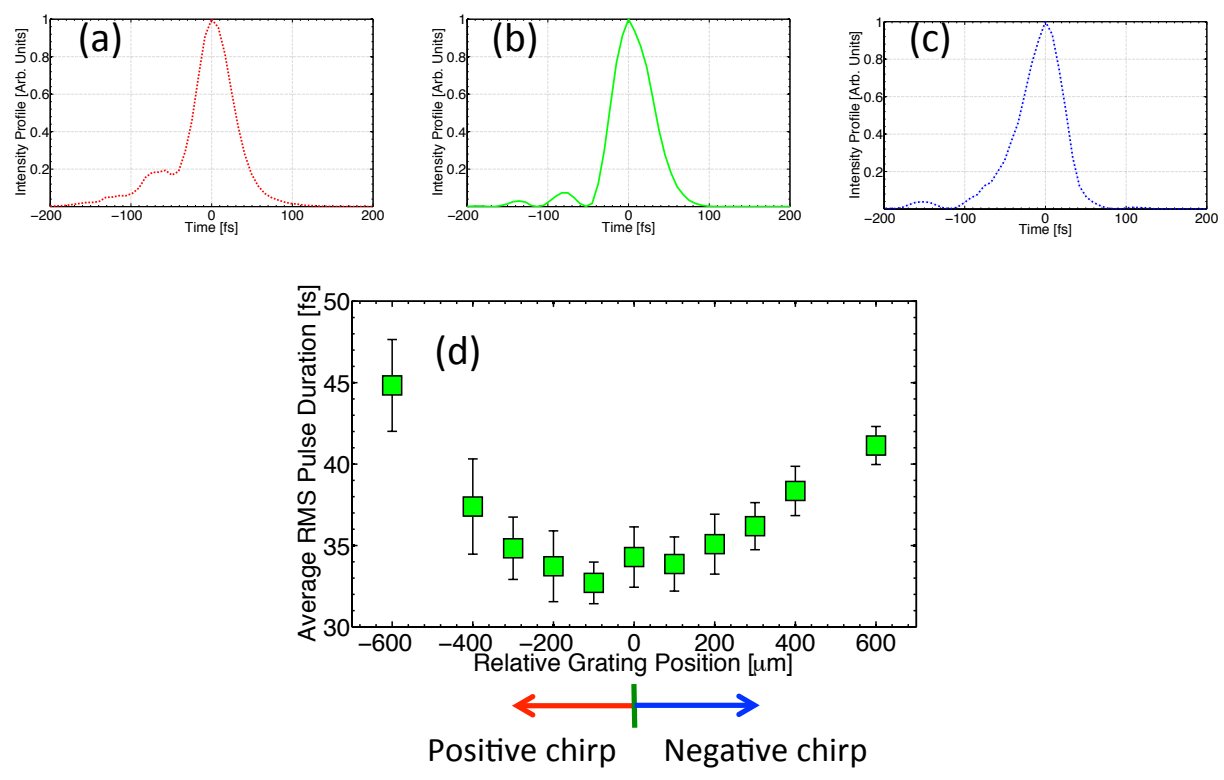

Figure 2. (Color online) Typical time-dependent intensity profile of the laser pulse for (a) positive chirps, (b) optimal compressor setting, and (c) negative chirps. Negative times corresponds to the leading edge of the pulse. (d) Average RMS pulse duration as a function of the relative grating position. 


\section{Experimental results}

For the first experiment, the laser beam delivered an average $1.8 \mathrm{~J}$ on target in a $34 \mathrm{fs}$ pulse, yielding an on-target peak intensity of $2.0 \times 10^{19} \mathrm{~W} / \mathrm{cm}^{2}$. A 3D-printed $5 \mathrm{~mm}$ single-stage gas cell was used as the target [21] with the cell filled with a mixed gas $(97.5 \%$ He and $2.5 \% \mathrm{~N}_{2}$ ) $[22,23]$. In the second experiment, a 25/75\% (reflection/transmission) $800 \mathrm{~nm}$ beamsplitter was present in the chamber which effectively reduced the average energy on target to $1.4 \mathrm{~J}$. A $3 \mathrm{~mm}$ single-stage gas cell was used with the cell filled with either the $2.5 \% \mathrm{~N}_{2}$ mixture or pure He depending on the parameter being scanned. For the first experiment, the electron densities were scanned from below injection threshold to significantly above threshold. Figure 3 shows typical electron spectra (generated using the mixed gas) corresponding to four of the density steps scanned. At the lower densities, quasimonoenergetic electron beams with low charge and divergence $(<10$ mrad full-angle) were generated. As the density increased, the wakefield cavity becomes overloaded with electrons and large-divergence, large energy spread beams are formed. Data from the first experiment are shown in Figs. 4 (a) - 4 (c) while Figs. 4 (d) and 5 show data from the second experiment. In Figs. 4 (a) and (b), the curved lines connecting the markers for each density scan are meant to serve as a visual aid only.
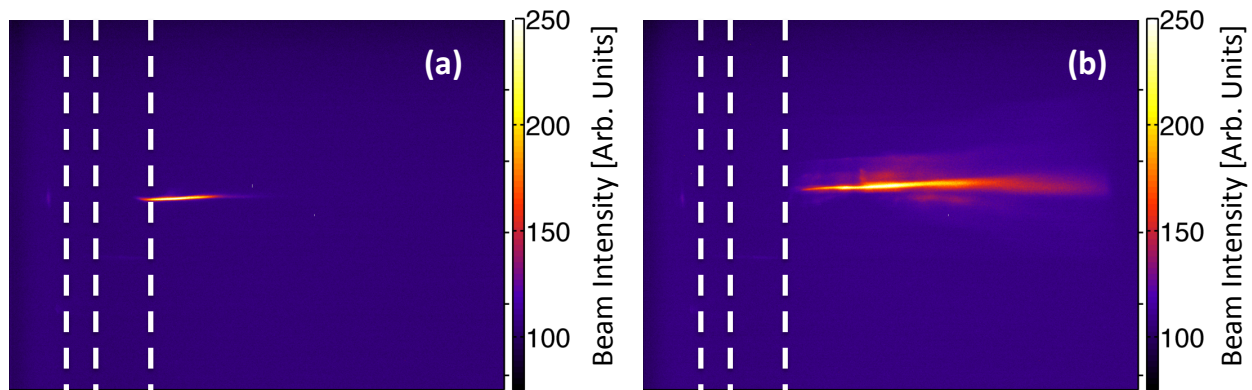

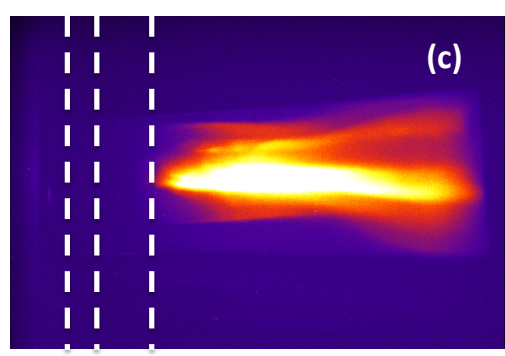

600400200

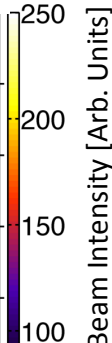

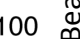

Electron Energy [MeV]

Figure 3. (Color online) Images of electron spectra from the ESPEC diagnostic for electron densities (a) 7.9 (b) 8.2 (c) 11 and (d) $13 \times 10^{18} \mathrm{~cm}^{-3}$. Quasimonoenergetic beams were generated at the lower densities while higher densities produced largedivergence beams with larger energy spread.

In Fig. 4 (a), the integrated electron charge on the ESPEC LANEX is plotted as a function of the relative grating separation for several different electron densities. The 
threshold density for electron injection for this data set was found to be $8.2 \times 10^{18} \mathrm{~cm}^{-3}$. This was verified by noting that a less than $5 \%$ decrease in the density resulted in a significant reduction in the electron beam charge and reproducibility at the shortest pulse duration and is reflected in Fig. 4 (a). The integrated electron charge was calculated by summing the total number of counts on the ESPEC LANEX which were then converted to charge via a cross-calibration technique using a FUJI BAS-MS image plate. The corresponding radiant energy produced by the betatron oscillations of the electrons from Fig. 4 (a) was calculated by directly summing the counts on the Andor CCD chip and is shown in Fig. 4 (b). The relationship between the radiant energy and the electron charge is plotted in Fig. 4 (c) and peak electron energies (defined as the energy containing the highest charge on the ESPEC LANEX) as a function of the relative grating separation are shown in Fig. 4 (d) for both gas types.

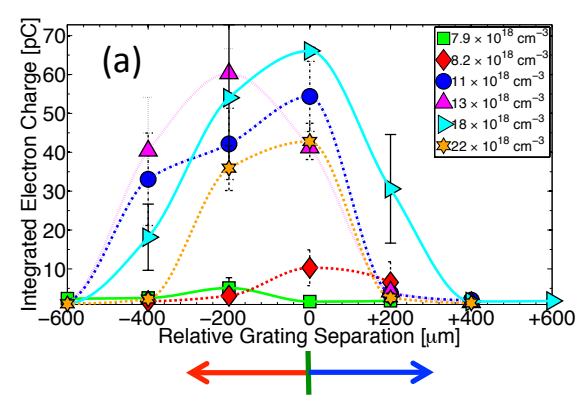

Positive chirp Negative chirp

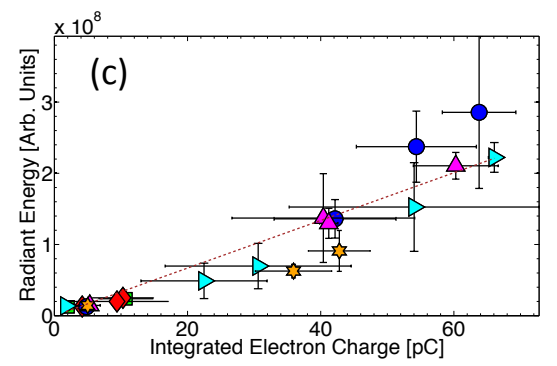

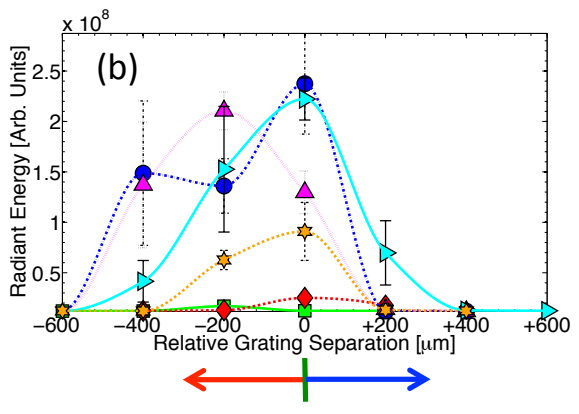

Positive chirp Negative chirp

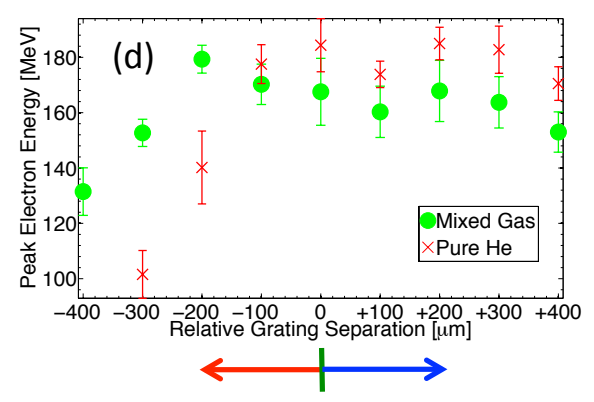

Positive chirp Negative chirp

Figure 4. (Color online) (a) Measurements of the integrated electron charge as a function of the relative grating separation for various electron densities (legend in inset). For similar separations, positive chirps produced more charge compared with negative chirps. (b) The corresponding effect on the radiant energy followed a similar trend. In both (a) and (b), the curved lines serve as a visual aid in connecting the data at each density step. (c) The radiant energy scaled linearly with the electron charge and was independent of the drive pulse parameters (dashed line denotes a linear fit to the data). (d) Peak electron energy as a function of compressor grating separation for the mixed gas (green circle) and pure He (red x). Error bars denote the standard error of the mean.

Figure 5 shows the X-ray spectra for both gas types as a function of the relative grating separation. Each spectrum represents an average constructed from five to ten shots using the method of single photon counting whereby the number of counts across 
the CCD chip is correlated with the photon energy incident on the camera $[24,25]$. Electron densities were nominally set to $6.5 \times 10^{18} \mathrm{~cm}^{-3}$ so that the integrated number of hits on the CCD camera remained within the single photon counting regime. In order to maintain injection at the larger grating separations (i.e., longer pulse durations), the density was increased up to a maximum of $10 \times 10^{18} \mathrm{~cm}^{-3}$. For the parameter range scanned, the probability of a double hit occurring based on Poisson statistics ranged between $0.5-4.8 \%$ on average. The algorithm used to construct the spectra is able to correct for predicted double hits for probabilities less than $15 \%$ [26].
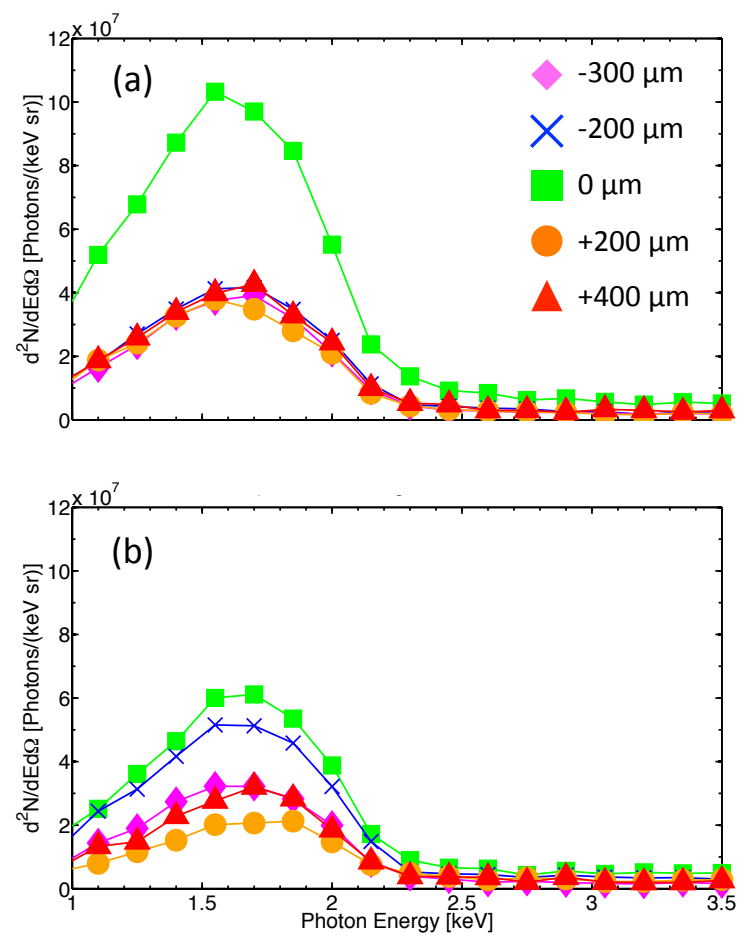

Figure 5. (Color online) (a) X-ray spectra for the $2.5 \% \mathrm{~N}_{2}$ mixed gas at different relative grating positions. The greatest number of photons was produced at the compressor zero setting (i.e., the shortest pulse duration with minimal frequency chirp). (b) X-ray spectra for pure He. A similar trend was observed for pure He as that for the mixed gas but with a larger spread in the photon flux around the peak energy.

\section{Discussion}

In general, strong asymmetries in both the electron charge and peak energy (and hence, the radiant energy) with respect to the relative grating position can be seen in Fig. 4 for all electron densities. Analysis of the pulse shape showed that positively chirped pulses exhibited a fast-rising leading edge compared with negatively chirped pulses. The fast-rising leading edge thus serves to enhance trapping of electrons as previously observed $[15,17]$. In contrast to previous studies, the shortest pulse duration (at compressor zero) still produced the highest number of accelerated electrons in the wakefield. From Fig. 2, we see that the slope of the leading edge of the pulse is 
comparable for positively chirped and optimally compressed pulses. As a result, the shorter pulse duration at compressor zero would drive the more efficient wakefield, resulting in the highest injected charge. In Ref. [18], an enhancement in the electron charge was observed for pulses with positive chirps at two different electron densities. In particular, the charge increase was more significant at the lower electron density. This was attributed to an enhancement in the forward Raman scattering (FRS) instability at lower densities. In our experiment, we scanned densities from below the injection threshold to significantly above threshold. A substantial enhancement in the charge was only observed at an electron density of $13 \times 10^{18} \mathrm{~cm}^{-3}$. Densities on either side this value shifted the optimal grating position for electron injection back to the compressor zero setting. At best, this enhancement is on par with the charge produced at other densities at the compressor zero setting.

Measurements of the peak electron energy showed that pulses on the negatively chirped side of compressor zero produced beams with higher peak energies than their counterparts on the positively chirped side. This trend was observed for both gas types as well. In addition, the peak energies obtained using negatively chirped pulses are comparable to those obtained at the compressor zero setting. This demonstrates the compromise that must be considered when optimizing the electron beam by changing the properties of the drive pulse. That is, positively chirped pulses with fast-rising leading edges generally injects more electrons into the bubble but at lower peak energies compared with negatively chirped pulses with a slow-rising leading edge.

Inspection of Figs. 4 (a) and (b) also show that the ratio between the radiant energy and electron charge is relatively constant and does not depend on the pulse characteristics. This is made even more evident in Fig. 4 (c) which shows the relationship between the integrated electron charge and the emitted X-rays to be fairly linear over a threefold change in the electron density. The dashed lined in Fig. 4 (c) is the best fit to the experimental data of the form $R=a \times Q$, where $R$ is the radiant energy, $Q$ is the integrated electron charge [pC], $a=9.8$. In Fig. 4 (c), no distinction has been made with regard to the pulse duration, pulse shape, or frequency chirp sign.

As shown in Fig. 5, the shape of the X-ray spectra and peak energy does not change appreciably as the compressor separation is varied (i.e., with changing pulse characteristics). For both gas types, the primary effect of changing the pulse characteristics is a change in the total number of photons in the spectra and, in particular, the number of photons around the peak energy. This change is primarily attributed to changes in the pulse duration and shape rather than the sign of the frequency chirp. As mentioned above, the electron density was also increased slightly in order to sustain injection at the longer pulse durations. However, the densities were changed by the same amount for both gas types at each compressor setting. Thus, differences in the spectra for the two gases cannot be a result of the density change alone since one would expect the total number of photons to increase with electron density regardless of the pulse duration [cf. Fig. 4 (c)].

For the mixed gas spectra, the standard error of the mean for the number of photons 
at the peak energy is $18 \%$ at the compressor zero and an average of $41 \%$ for the other settings. For the pure He spectra, the error is $30 \%$ at the compressor zero and an average of $51 \%$ for the other settings. Even with the relatively large error in the flux at the non-optimal grating separations, the shortest pulse duration still produced the highest photon flux for both gas types, with the flux generally being higher for the mixed gas as a result of the higher average electron charge created in the ionization injection process. Ionization injection also produced a smaller variation in the synchrotron spectra compared with self injection. The smaller spread in the X-ray spectra for the mixed gas can be explained by considering the differences in the injection process between the pure He and mixed gas. The majority of electrons in the mixed gas (resulting from nitrogen) are freed near the peak of the laser pulse. Thus, pulses with asymmetric rise/fall times are not likely to significantly affect the ionization and injection process for the mixed gas. For the pure He case, changes in the pulse characteristics can enhance laser self-focusing and self-steepening, both of which plays a larger role in the self-injection process.

\section{Conclusion}

Our experiments show that altering the laser pulse properties in a typical Ti:Sapphire CPA system can affect the electron injection process which in turn directly affects the emitted radiation. Positively chirped pulses with a fast-rising leading edge led to an increase in the electron charge compared with negatively chirped pulses with a slowrising leading edge but produced electron beams with lower peak energies. The X-ray flux trend matched that of the integrated electron charge. The emitted radiation also scaled linearly with the electron charge over a threefold change in the electron density, independent of the laser pulse characteristics. X-ray spectra showed that the $2.5 \%$ mixed gas produced more photons than the pure He and had a smaller spread in the spectra due to ionization injection. In all cases, however, optimal pulse characteristics generally produced the highest number and peak energy of injected electrons and photons.

\section{Acknowledgments}

This work is supported by the U.S. Department of Energy/National Nuclear Security Administration grant DE-NA0002372 and the National Science Foundation Career grant 1054164 .

\section{References}

[1] T. Tajima and J.M. Dawson, Phys. Rev. Lett. 43267 (1979).

[2] S.P.D. Mangles et al., Nature (London) 431535 (2004).

[3] C.G.R. Geddes et al., Nature (London) 431538 (2004).

[4] J. Faure et al., Nature (London) 431541 (2004).

[5] W.P. Leemans et al., Nature Phys. 2696 (2006).

[6] S. Corde et al., Rev. Mod. Phys. 85, (2013).

[7] S. Kneip et al., Nat. Phys. 6, 980 (2010). 
[8] S. Kneip et al., Appl. Phys. Lett. 99, 093701 (2011).

[9] V. Malka et al., Science 2985598 (2002).

[10] A. Pukhov and J. Meyer-ter-Vehn, Appl. Phys. B 74355 (2002).

[11] A. G. Khachatryan, F. A. van Goor, and K.-J. Boller, Phys. Rev. E. 70, 067601 (2004).

[12] S. Afhami, and E. Eslami, Phys. Plasmas 21, 063108 (2014).

[13] V. B. Pathak et al., New J. Phys. 14, 023057 (2012).

[14] D. S. Dodd and D. Umstadter et al., Phys. Plasmas 8, 3531 (2001).

[15] W. P. Leemans et al., Phys. Rev. Lett. 89, 174802 (2002).

[16] C. B. Schroeder et al., Phys. Plasmas 10, 2039 (2003).

[17] S. P. D. Mangles et al., Phys. Rev. ST Accel. Beams 15, 011302 (2012).

[18] B. S. Rao et al., Phys. Rev. ST Accel. Beams 16, 091301 (2013).

[19] S. Y. Kalmykov et al., Phys. Plasmas 22, 056701 (2015).

[20] K. W. DeLong and R. Trebino, J. Opt. Soc. Am. A 11, 9 (1994).

[21] M. Vargas et al., Appl. Phys. Letters 104, 56704 (2014).

[22] C. McGuffey et al., Phys. Rev. Lett. 104, 025004 (2010).

[23] A. Pak et al., Phys. Rev. Lett. 104, 025003 (2010).

[24] S. Fourmaux et al., New J. Phys. 13, 033017 (2011).

[25] C. Fourment et al., Rev. Sci. Instrum. 80, 083505 (2009).

[26] K. Behm, T. Z. Zhao et al., Phys. Plasma. Control. Fusion (2016). 